Ann. Zootech. I979, 28 (4), 393-406.

\title{
Estimation de la composition chimique du mouton adulte
}

\author{
Françoise DOIZE, R. de WILDE * et R. PAQUAY
}

avec la collaboration technique de Marie-Antoinette Bouckoms et D. CroostERmans

Laboratoire de Physiologie Animale, Facultés Universitaires, N.-D. de la Paix, rue de Bruxelles 6I, B-50oo Namur, Belgique

\section{Résumé}

En vue d'élaborer des formules permettant d'estimer la composition chimique de moutons adultes montrant une très grande variabilité de poids et de niveau d'engraissement, I 3 brebis et 2 béliers Suffolk, âgés d'au moins 2 ans et demi, ont été abattus et analysés après la détermination de l'espace de diffusion de l'eau tritiée. Ils avaient auparavant été soumis, pendant cles périodes de temps prolongées, à des conditions alimentaires très variables.

Le poids vif des moutons variait de 43,5 à $93,9 \mathrm{~kg}$ et la teneur en graisses du poids vide sans laine, de 7,2 à 48,2 p. 100 .

La différence de poids entre les animaux gras (plus de $60 \mathrm{~kg}$ de poids vide sans laine) et les autres est constituée à raison de $60 \mathrm{p}$. Ioo par de la graisse.

Lorsque le poids vide sans laine est connu, il est possible d'estimer avec une bonne approximation la composition chimique du corps des animaux.

En moyenne, l'espace de diffusion de l'eau tritiée surestime de 5,7 p. too le volume total d'eau corporelle. Cette différence n'est pas influencée par l'état d'engraissement.

A partir du poids vif (PV, en $\mathrm{kg}$ ) et de l'espace de diffusion de l'eau tritiée (ED, en 1) les équations suivantes ont été calculées :

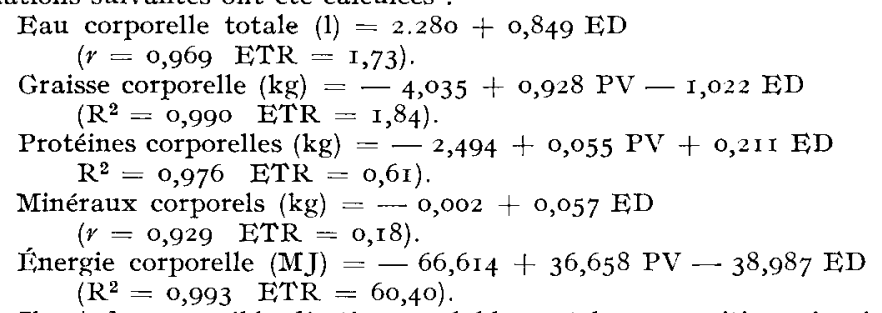

Il est donc possible d'estimer valablement la composition " in vivo " de moutons adultes à partir du poids vif et de l'espace de diffusion de l'eau tritiée.

\footnotetext{
* I,aboratorium voor diervoedingleer, Rijksuniversiteit Gent, Merelbeke, Melgique.
} 


\title{
Introduction
}

Dans ses méthodes d'évaluation des espèces domestiques, le zootechnicien a toujours été attentif aux modifications de la composition chimique des animaux lors de la croissance ou de l'engraissement. Il s'est efforcé d'estimer. sinon de déterminer, les changements provoqués par l'alimentation dans les dépôts corporels, principalement de graisses et de substances azotées.

Le mouton est un animal largement étudié dans ce domaine, mais la plupart des publications traitant de la composition chimique de cette espèce et de son estimation ont été réalisées à partir d'animaux en croissance ou adultes ne présentant qu'un éventail limité de variations pondérales. Les études réalisées sur des groupes d'ovins adultes montrant une très grande variabilité de poids et pouvant atteindre un poids vif élevé sont rares.

Dans le cadre de nos recherches sur le contrôle à long terme des ingestions volontaires, nous provoquons chez le mouton des variations considérabies de poids. Nous devons être capables d'estimer régulièrement l'état d'engraissement des animaux, aussi avons nous été amenés à mettre au point une méthode permettant d'estimer " in vivo" la composition corporelle du mouton.

Deux revues bibliographiques (DUMONT, I958; RoBELIN, I973) et divers articles publiés sur ce sujet lors de la dernière décennie et relatifs au mouton

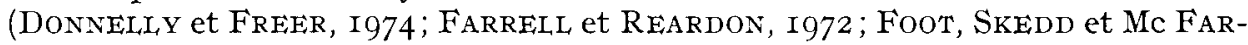
IANE, I979; HoUseman, Robinson et Fraser, I978; KEENAN, Mc Manus et FREER, I969; SEARLE, I970; SMITH et SykES, I974) nous ont incité à utiliser la technique de l'espace de diffusion de l'eau tritiée. Pour estimer la composition de nos animaux, nous avons ensuite utilisé les formules données par ces auteurs et permettant de déterminer les teneurs en eau, en graisses, en protéines, en minéraux et en énergie des organismes à partir du poids vif et de l'espace de diffusion. Nous avons, pour chaque animal et pour chaque constituant corporel, obtenu des résultats parfois très différents selon les formules utilisées. Nous avons en conséquence été amenés à abattre un groupe d'animaux afin de déterminer la composition corporelle et mettre au point nos propres équations.

Les résultats relatifs à la composition corporelle et à l'estimation de cette composition sont décrits dans cet article.

\section{Matériel et méthodes}

\author{
Animaux et alimentation
}

Quinze moutons, treize brebis et deux béliers, de race Suffolk ont été utilisés. Quelques informations générales relatives à ces animaux et à leurs conditions préalables d'alimentation et d'évolution pondérale sont données dans le tableau $\mathrm{I}$.

Les moutons étaient âgés d'au moins deux ans et demi et montraient une conformation zootechnique moyenne pour des animaux de cette race dans notre pays. Ils avaient été soumis auparavant à des conditions alimentaires très variables 


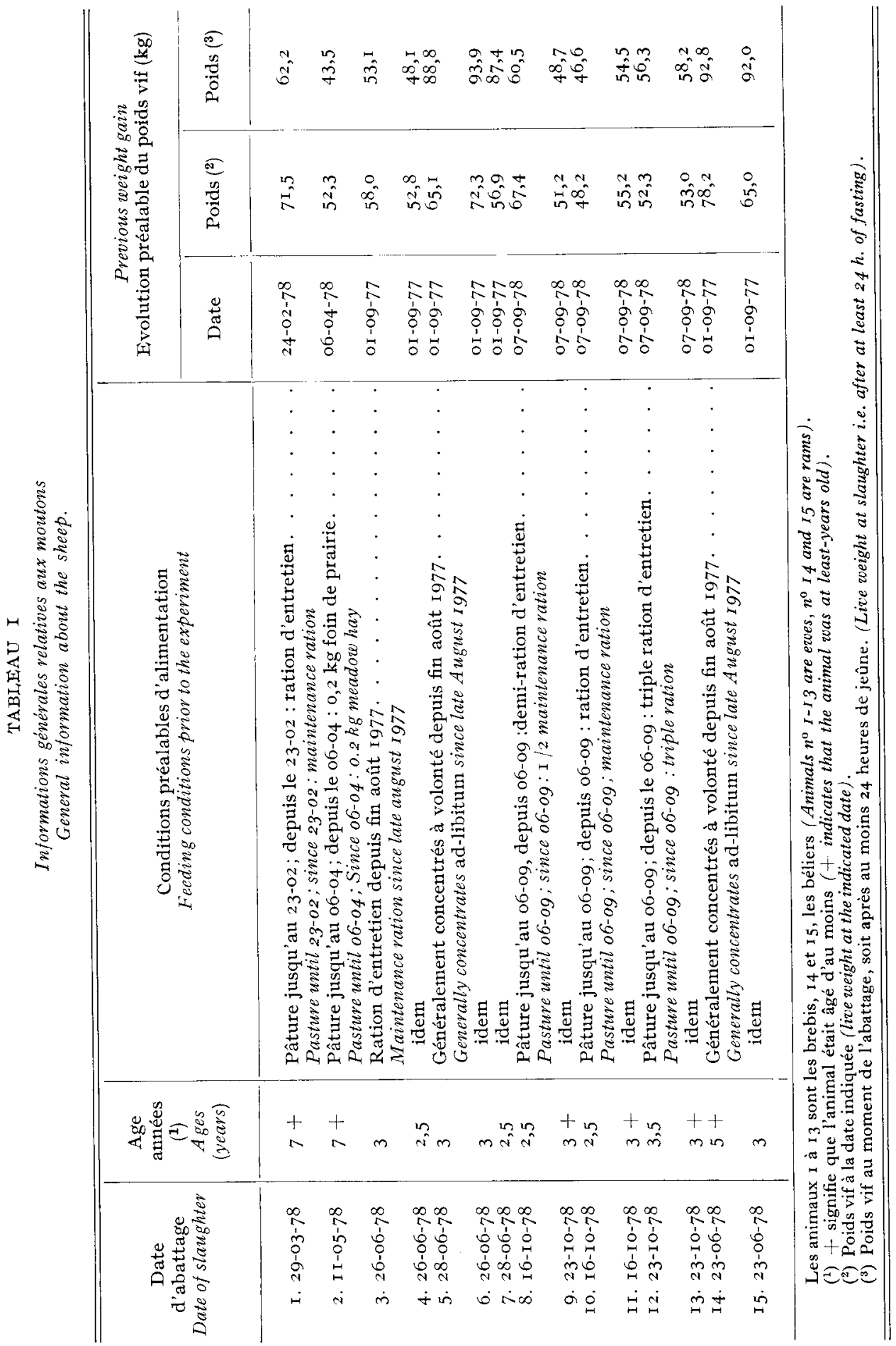


allant de l'ingestion illimitée de concentrés pendant de nombreux mois à une stricte sous-alimentation. Il s'en était suivi de fortes modifications du poids vif.

\section{Abattage et analyse des animaux}

Les animaux, après avoir subi le test d'estimation de l'espace de diffusion de l'eau tritiée, soit après au moins 24 heures de mise à jeun, sont pesés à Ioo g près. Ils sont tondus et la laine est pesée et échantillonnée. Une tranquillisation au Rompun (dérivé de la Thiazine) et l'injection intraveineuse d'héparine (en vue de faciliter la récolte du sang) sont réalisées. Une dose de 600 à I $200 \mathrm{mg}$ de pentobarbital sodique est administrée et les vaisseaux du cou sont sectionnés. Le sang et le contenu digestif sont recueillis, pesés et échantillonnés. Les abats (organes internes, peau, graisses, tête, extrémités des membres) sont séparés de la carcasse et, de même que les deux moitiés de celle-ci, sont pesés.

Les abats et la moitié de la carcasse sont autoclavés dans des récipients séparés, pendant I 5 heures à $125^{\circ} \mathrm{C}$ (DE WILDE, I977). Les différents composants (y compris les os et les dents) sont ramollis. Ils peuvent être ainsi facilement homogénéisés et échantillonnés.

La teneur en matière sèche du contenu digestif est déterminée par dessication à l'étuve à Ioo $^{\circ} \mathrm{C}$ pendant 24 heures. Ia laine, le sang, la carcasse et les abats sont analysés par les méthodes classiques : 1yophilisation pour la matière sèche, méthode de Kjeldahl pour les protéines (azote $\times 6,25$ ), extraction à l'éther pour les graisses, incinération au four pour les matières minérales.

\section{Détermination de l'espace de diffusion de l'eau tritiée}

Le jour avant la mesure de l'espace de diffusion de l'eau tritiée, les aliments sont distribués aux heures habituelles (Io $h$ pour le foin, II $h$ zo pour les concentrés). Les refus sont retirés à I 6 h et 1 'eau de boisson est supprimée le lendemain matin à $9 \mathrm{~h}$, soit au moment de l'injection de l'eau tritiée. Cette injection est précédée d'une première prise de sang par ponction jugulaire, récoltée dans un tube hépariné. Un $\mathrm{ml}$ de la solution stock d'eau tritiée $(275 \mu \mathrm{Ci}$ diulés dans de l'eau physiologique) est dilué dans 2,5 à $3 \mathrm{ml}$ d'eau physiologique et injecté dans la jugulaire.

Sept heures plus tard, une deuxième prise de sang est réalisée dans la jugulaire opposée. Les animaux sont pesés à roo g près; les urines éliminées depuis l'injection sont pesées et échantillonnées.

Les échantillons de sang sont centrifugés et I ml de plasma est placé dans une fiole contenant $9 \mathrm{ml}$ de liquide de scintillation (Lumagel de Lumac). Le comptage (en 3 reprises au moins) est réalisé après un séjour de $\mathrm{r} 2$ heures à 1'obscurité. Ensuite, Ioo $\mu \mathrm{Ci}$ de toluène tritié sont ajoutés à chaque échantillon et un second comptage est réalisé.

Les échantillons d'urine sont centrifugés et traités de la même manière que le plasma.

L'espace de diffusion de l'eau tritiée est calculé à partir du nombre de dpm injectés et du nombre de $\mathrm{dpm}$ contentis dans chaque $\mathrm{m} 1 \mathrm{de}$ plasma et réellement dus à l'injection. Ces nombres sont obtenus en déterminant l'efficience de comptage de chaque échantillon (par le toluène), en retirant des valeurs obtenues le soir 
( $2^{\mathrm{e}}$ prise de sang) celles mesurées le matin ( $\mathrm{I}^{\mathrm{re}}$ prise de sang) et en apportant une correction (I.0989) pour la teneur en matière sèche du plasma (BL,UNT, I975).

Les pertes de marqueur par les urines peuvent être déduites du nombre total de đpm injectés.

\section{Résultats}

\section{Constituants corporels et composition chimique}

Le poids vif des animaux au moment de l'abattage (tableau I) varie entre 43,5 et $93,9 \mathrm{~kg}$ avec une moyenne de $65,8( \pm \mathrm{I} 9,2) \mathrm{kg}$.

Le contenu digestif pèse entre 5,2 et $9,9 \mathrm{~kg}$ avec une moyenne de $7,4 \pm \mathrm{I}, 5 \mathrm{~kg}$. Ein moyenne, il représente $I I, 2 \mathrm{p}$. Ioo du poids vif et son eau constitue de $\mathrm{II}_{1}, 8$ à 28, 4 p. roo de l'eat corporelle totale ( $77,9 \pm 5$, o en moyenne).

La laine recueillie pèse entre 0,8 et $3,7 \mathrm{~kg}$ par animal avec une moyenne de $2,0 \mathrm{~kg}$. Les protéines constituent l'élément de base de la laine $(63, \mathrm{I} \pm 3,8 \mathrm{p}$. Ioo en moyenne de la laine totale). La teneur en eau est de 6,2 à I3,7 p. Ioo avec une moyenne de ro,o $( \pm 2,2)$ p. Ioo et cette eau représente en moyenne $0,56 \mathrm{p}$. Ioo de l'eau corporelle totale.

Selon les animaux, 2,5 à 4,4 kg de sang sont recueillis ( $3,2 \pm 0,5 \mathrm{~kg}$ en moyenne). Ce sang ne constitue donc en moyenne que 4,9 p. Ioo du poids vif soit une valeur inférieure à celle généralement donnée pour le volume sanguin total (7 p. I00).

La teneur en matière sèche du sang varie de 8,3 à $2 \mathrm{I}, 9 \mathrm{p}$. Ioo avec une moyenne de $15,9 \pm 3,8$ p. roo. Cette matière sèche est essentiellement constituée de substances azotées car matières grasses et minéraux réunis représentent moins de I p. Ioo du sang total.

Les carcasses pèsent entre 15,8 et $58,7 \mathrm{~kg}$ avec une moyenne de $32,7 \pm \mathrm{I} 2,6 \mathrm{~kg}$, soit de 36,3 à $57,2 \mathrm{p}$. Ioo du poids vif $(48,6 \pm 5,6 \mathrm{p}$. Ioo en moyenne). Les teneurs en eau (37,0 à 66,3 p. IO0, 52,0 $\pm 7,4$ en moyenne), en graisses $(8,7$ à 48,8 p. IoO, $27,0 \pm \mathrm{I} 0,0$ en moyenne), en protéines (I 2,0 à I 9,6 p. IOO, I $6,6 \pm 2$, I en moyenne) et en énergie $(7,3$ à $2 \mathrm{r}, 3 \mathrm{MJ} / \mathrm{kg}, \mathrm{I} 3,4 \pm 3,7$ en moyenne) y sont très variables. Ajoutons qu'en moyenne les subtances azotées des carcasses ne forment que $54 \mathrm{p}$. Ioo des substances azotées corporelles totales du corps vide.

Les abats constituent de I3, I à $30, \mathrm{I} \mathrm{kg}(20,5 \pm 6,2$ en moyenne), soit de 28 , I à 35,9 p. Ioo du poids vif (3I,I $\pm 2,5$ en moyenne). La composition chimique moyenne est proche de celle des carcasses mais avec des extrêmes plus accentués pour les différents constituants. Il faut d'ailleurs noter qu'il n'est pas toujours facile de réaliser une nette séparation entre la carcasse et les abats, notamment au niveau des graisses sous-cutanées et abdominales.

Diverses autres valeurs relatives à la composition chimique des animaux sont données dans le tableau 2. La graisse est l'élément le plus variable du corps vide sans laine $(7,2$ à 48,2 p. IOo) mais on notera aussi que les teneurs en protéines et en minéraux sont loin d'être constantes. S'il est exprimé sur la base de la matière sèche, le corps vide sans laine se compose de 23,8 à $77,4 \mathrm{p}$. Ioo de graisses $(54,9 \pm \mathrm{r} 4,4 \mathrm{p}$. Ioo en moyenne), de I 7,5 à $56,3 \mathrm{p}$. Ioo de protéines $(34,0 \pm \mathrm{Io}, 2)$ et de 4,0 à $\mathrm{I} 4,9 \mathrm{p}$. Ioo de minéraux $(9,0 \pm 3, \mathrm{I})$. Ces modifications, liées à celles du poids de l'animal, font que la quantité totale d'énergie contenue dans la carcasse et les abats varie de 209 à I $8 \mathrm{I} 4 \mathrm{MJ}$. 


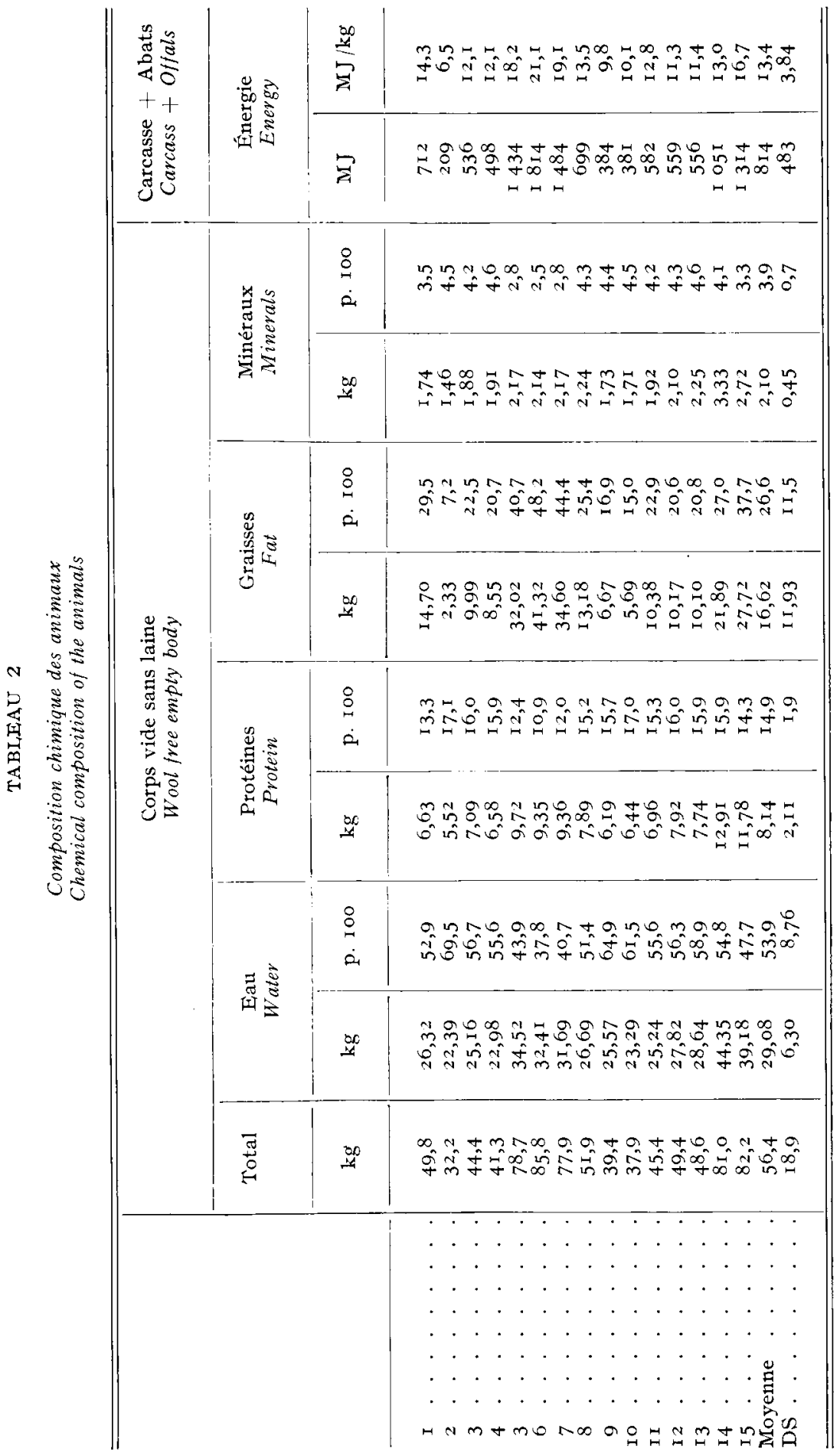




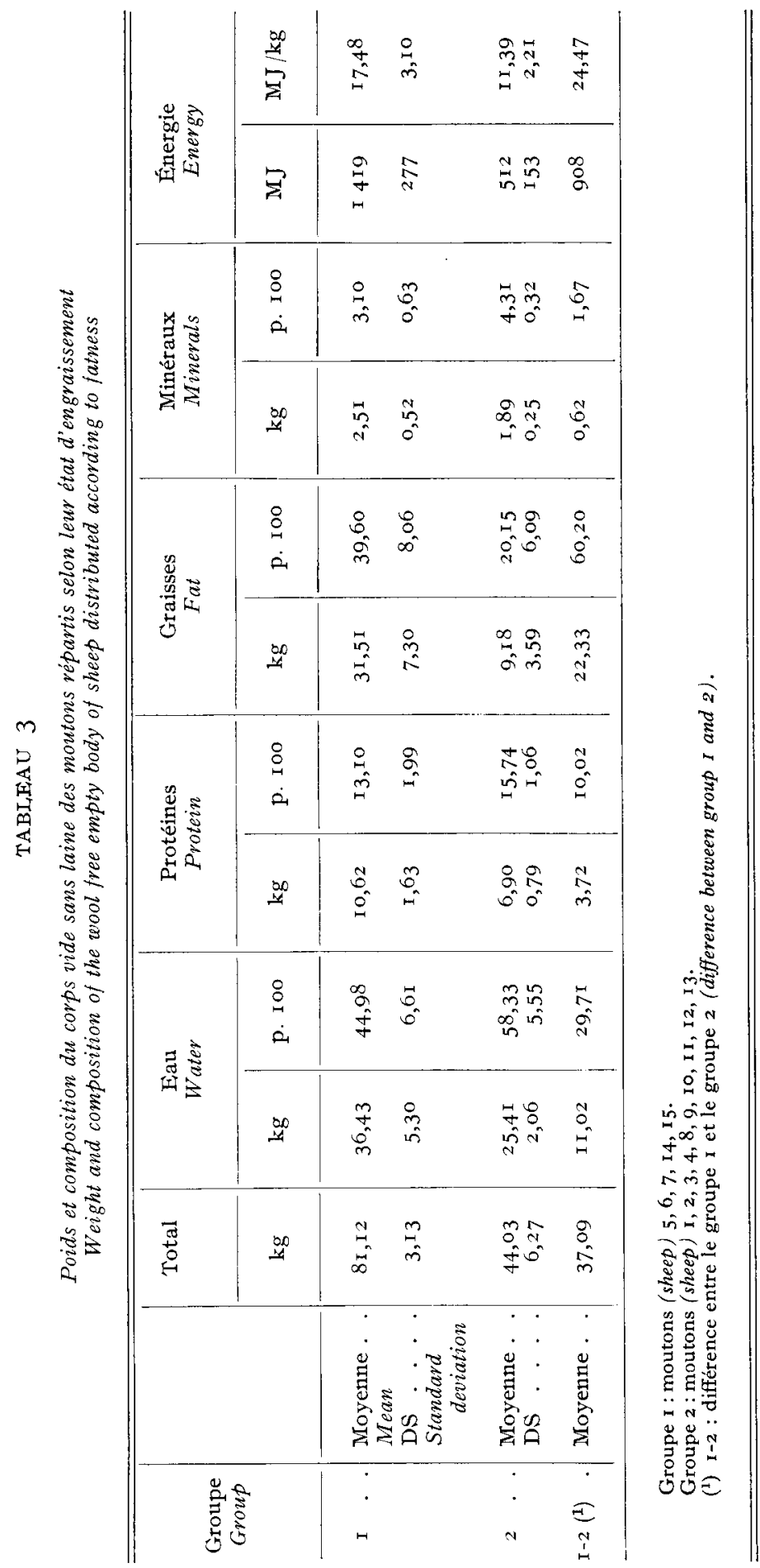




\section{Effets de l'engraissement sur la composition chimique du corps}

Les animaux ont, selon leur état d'engraissement, été divisés en deux groupes : gras (groupe $\mathrm{I}$, plus de $60 \mathrm{~kg}$ de poids vide sans laine, 5 moutons) et non gras (groupe 2 , moin de $60 \mathrm{~kg}$, Io moutons). Les valeurs moyennes pour le poids vide sans laine et sa composition ainsi que les différences entre les groupes sont données dans le tableau 3 .

On observe que les animaux gras présentent un taux de graisses double de celui des animaux non gras et, la différence de poids aidant, la quantité d'énergie corporelle est trois fois plus grande chez les animaux gras. L'écart entre les teneurs en graisses est compensé principalement par un écart inverse entre les teneurs en eau, mais aussi en protéines et en minéraux.

Si on considère la différence de poids entre les deux groupes d'animaux, on constate que cette différence est constituée à raison de 60 p. Too par de la graisse et que les proportions d'eau, de protéines et de minéraux sont nettement inférieures aux teneurs corporelles de ces constituants, même chez le mouton gras.

\section{Relations entre les substances chimiques et le poids vide sans laine}

Les coefficients de corrélation entre le poids vide sans laine des animaux et les substances corporelles sont donnés dans le tableau 4 .

\section{TABLEAU 4}

Coefficients de corrélation entre le poids vif vide sans laine et les substances corporelles Coefficients of correlation between wool free empty body weight and body components

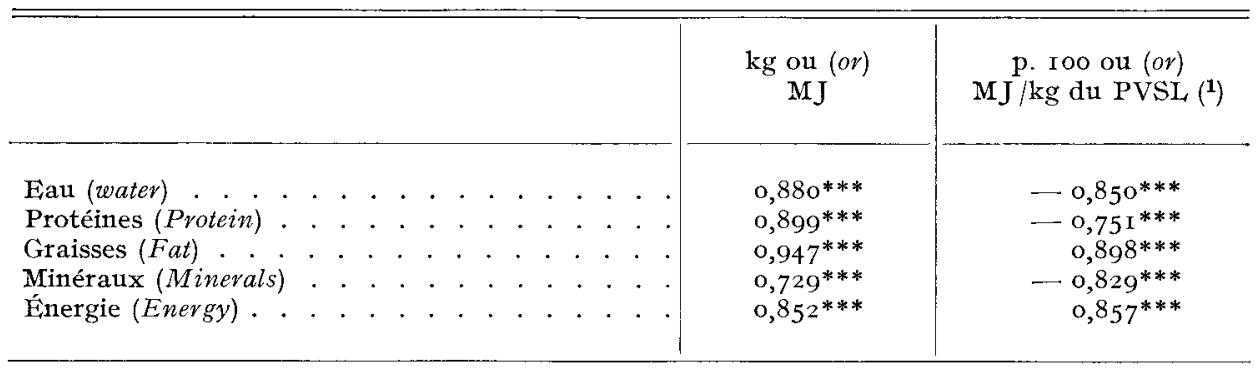

*** $P<0,001$.

(1) PVSL $\stackrel{0,001}{=}$ wool free empty body weight.

Il en ressort que pour l'ensemble des animaux, il existe une relation étroite entre le poids vide sans laine et la composition chimique. Lorsque ce poids est connu, il est donc possible d'estimer avec une bonne approximation la composition chimique du mouton adulte quel que soit son niveau d'engraissement. Les équations suivantes, avec $x=$ le poids vif vide sans laine (en $\mathrm{kg}$ ) et $y=$ les constituants corporels (en $\mathrm{kg}$ ou en $\mathrm{M}$ ), ont été obtenues :

$$
\text { eau } \begin{aligned}
(\mathrm{kg}): y & =0,29 x+\mathrm{r} 2,5 \mathrm{I} \\
(r & =0,880 \quad \text { ETR }=3, \mathrm{II}),
\end{aligned}
$$




$$
\begin{aligned}
& \text { protéines }(\mathrm{kg}): y=0 . \operatorname{Io} x+2,46 \\
& (r=0.899 \quad \mathrm{E} \text { 'TR }=0,96) \text {. } \\
& \text { graisses }(\mathrm{kg}): \begin{array}{l}
y=0.60 x-\mathrm{I} 7, \mathrm{I} 8 \\
(r=0.947 \quad \mathrm{ETR}=3,96) .
\end{array} \\
& \text { énergie }(\mathrm{MJ}): y=24.50 x-567,47 \\
& (r=0.957 \quad \mathrm{ETR}=\mathrm{I} 45,63) .
\end{aligned}
$$

\section{Espace de diffusion de l'eau tritiée et eau corporelle}

Étant donné que nous avons eu des ennuis techniques lors de la détermination de l'espace de diffusion de l'eau tritiée chez deux brebis (Io et I3) et que les résultats obtenus pour ces animaux sont dès lors sujets à caution, ils sont éliminés

\begin{tabular}{|c|c|c|c|c|}
\hline & & \multicolumn{3}{|c|}{$\begin{array}{l}\text { Eau corporelle totale (p. Ioo du poids vif) } \\
\text { Total body water }(p . \text { Ioo of live weight })\end{array}$} \\
\hline & & $\begin{array}{c}\text { Espace de diffusion } \\
\text { Water space }\end{array}$ & $\begin{array}{l}\text { Déterminée } \\
\text { Determined }\end{array}$ & $\begin{array}{l}\text { Différence } \\
\text { Difference }\end{array}$ \\
\hline ...... . & . & 59,65 & $57, \mathrm{I} 4$ & $2,5 \mathrm{I}$ \\
\hline . . . . . & .. . & 79,95 & 72,22 & 7,73 \\
\hline ....... & . . . & 64,26 & 60,52 & 3,74 \\
\hline ..... & .. . & 67,70 & 57,76 & 9,94 \\
\hline 5. & . . & 50,66 & 45,74 & 4,92 \\
\hline ..... & . . . & $4 \mathrm{I}, 66$ & $39,9 \mathrm{I}$ & $\mathrm{I}, 75$ \\
\hline . . . . . & . . & 52,00 & 43,91 & 8,09 \\
\hline ...... . & . & 62,56 & 53,23 & 9,33 \\
\hline $9 \cdot$. & . . & $67, \mathrm{x} 7$ & 66, I 4 & $\mathrm{I}, 03$ \\
\hline II . . & . . & $63,7^{6}$ & 57,49 & 6,27 \\
\hline $12 \ldots$ & . . . . & 64,94 & 57,61 & 7,33 \\
\hline $14 \ldots \ldots$ & . . . . & 61,57 & 56,60 & 4,97 \\
\hline & . . . . & 56,01 & $4^{8,74}$ & 7,27 \\
\hline Moyenne (Average) $\ldots$ & . . . . & 60,91 & $55, \mathrm{I} 5$ & 5,76 \\
\hline DS (Standard deviation) . & . . . . & 9,43 & 9,93 & 2,87 \\
\hline
\end{tabular}
pour l'interprétation des résultats de l'espace de diffusion de l'eau tritiée.

TABLEAU 5

Eau corporelle des animaux

Body water of the animals

Les résultats individuels et moyens relatifs à 1'eau corporelle des animaux sont donnés dans le tableau 5. Dans le calcul de 1'espace de diffusion, il n'est pas tenu compte des pertes de tritium par les urines.

Dans ces conditions, l'espace de diffusion de l'eau tritiée surestime, en moyenne, 1'eau corporelle totale de 5,7 p. roo.

$\mathrm{Si}$, comme précédemment, nous divisons les moutons en gras (groupe I, tabl. 3) et non gras (goupe 2), la surestimation de l'eau corporelle par l'espace de diffusion est en moyenne de $5,40 \pm 2,47$ et $5,99 \pm 3,24 \mathrm{p}$. Ioo pour les groupes I et 2 . L'état d'engraissement n'a done pas d'effet apparent sur la valeur de la surestimation. 
Pendant les 7 heures séparant l'injection de l'eau tritiée de la deuxième prise de sang, II des I 3 moutons ont perdu en moyenne dans les urines $0,42( \pm 0,27)$ p. Ioo avec des valeurs extrêmes de $0, x$ I et 0,8 I p. Ioo du tritium injecté. Cette valeur ne représente donc qu'une faible proportion de la surestimation totale de l'eau corporelle par 1'espace de diffusion. Elle peut être négligée.

\section{Estimation in vivo de la composition corporelle}

Les coefficients de corrélation, simples et multiples obtenus entre, d'une part le poids vif (PV) ou (et) l'espace de diffusion de l'eau tritiée (F,D) et d'autre part les constituants corporels sont donnés dans le tableau 6 .

\section{TABLEAU 6}

Coefficients de corrélation, simples et multiples, entre les constituants corporels, le poids vif et l'espace de diffusion de l'eau tritiée.

Simple and multiple coefficients of correlation between body components, live weight and tritiated water space

\begin{tabular}{|c|c|c|c|c|c|c|}
\hline & & & Poids vif & \multicolumn{2}{|c|}{$\begin{array}{l}\text { Espace de diffusion } \\
\text { Tritiated water space }\end{array}$} & \multirow{2}{*}{$\begin{array}{c}\text { Poids vif }+ \\
\text { espace } \\
\text { de diffusion } \\
\text { Live weight } \\
+ \text { tritiated } \\
\text { water space } \\
(\mathrm{kg} \text { et } l) \\
\mathbf{R}^{\mathbf{2}}\end{array}$} \\
\hline & & & & $\begin{array}{c}(l) \\
r\end{array}$ & $\underset{r}{(\text { p. Ioo PV) }}$ & \\
\hline $\begin{array}{l}\text { Eau corporelle totale } \\
\text { Total body water }\end{array}$ & $\begin{array}{l}\mathrm{kg} \cdot \cdot \dot{\mathrm{PV}} \\
\mathrm{p} \cdot \mathrm{I} \text { оo }\end{array}$ & $\cdot$ & $\begin{array}{r}0,845^{* * *} \\
-0,826^{* * *}\end{array}$ & $\begin{array}{l}0,970^{* * *} \\
-0,47 \mathbf{I}\end{array}$ & $0,953^{* * *}$ & $0,94^{2 * * *}$ \\
\hline $\begin{array}{l}\text { Graisse corporelle } \\
\text { Body fat }\end{array}$ & $\begin{array}{l}\mathrm{kg} \cdot \cdot \dot{\mathrm{PV}} \\
\mathrm{p} \cdot \mathrm{I} \text {. }\end{array}$ & . & $\begin{array}{l}0,933^{* * *} \\
0,791 * *\end{array}$ & $\begin{array}{r}0,614 \\
-0,348\end{array}$ & $-0,976^{* * *}$ & $0,984 * * *$ \\
\hline $\begin{array}{l}\text { Protéines corporelles } \\
\text { Body protein }\end{array}$ & $\begin{array}{l}\mathrm{kg} \cdot \text {. } \\
\mathrm{p} . \text { Ioo } \dot{\mathrm{PV}}\end{array}$ & $\dot{\cdot}$ & $\begin{array}{l}0,85^{* * * *} \\
-0,4 \mathrm{I} 2\end{array}$ & $\begin{array}{l}0,826 * * * \\
0,026\end{array}$ & 0,7 I $5^{* *}$ & $0,954 * * *$ \\
\hline $\begin{array}{l}\text { Minéraux corporels } \\
\text { Body minevals }\end{array}$ & $\begin{array}{l}\mathrm{kg} \cdot \text {. } \\
\mathrm{p} \text {. I } \mathrm{P} \text {. }\end{array}$ & $\dot{\cdot}$ & $\begin{array}{r}0,793^{* * *} \\
-0,793^{* * *}\end{array}$ & $\begin{array}{l}0,930^{* * *} \\
-0,444\end{array}$ & - 0,096 & $0,864 * * *$ \\
\hline $\begin{array}{l}\text { Énergie corporelle } \\
\text { Body energy }\end{array}$ & $\begin{array}{l}\text { Meal. . . } \\
\mathrm{kcal} / \mathrm{kg} .\end{array}$ & $\dot{\cdot}$ & $\begin{array}{l}0,94 I^{* * *} \\
0,819^{* * *}\end{array}$ & $\begin{array}{l}0,630^{*} \\
0,360\end{array}$ & $\rightarrow 0,023$ & $0,989^{* * *}$ \\
\hline
\end{tabular}

Degré de signification (Degree of significance) : * ${ }^{*}=p<0,05{ }^{* *}=p<0,0 \mathrm{I} ; *^{* *}=p<0, \mathrm{oor}$.

A partir de ED, seul ou avec le poids vif, il est donc possible d'estimer in vivo, avec une bonne approximation la composition chimique du mouton adulte.

L'introduction du poids vif dans l'équation permettant de calculer l'eau corporelle totale à partir de ED n'améliore pas la valeur de l'estimation; l'équation suivante est retenue :

Eau corporelle totale $(\mathrm{kg})=2,280+0,849 \mathrm{ED}(l)$

$$
(r=0,969, \mathrm{E} \text { TR }=\mathrm{I}, 73)
$$


La prise en considération du poids vif permet au contraire d'améliorer l'estimation des graisses corporelles:

Graisses corporelles $(\mathrm{kg})=-4,035+0,928 \mathrm{PV}(\mathrm{kg})-\mathrm{I}, 022 \mathrm{ED}(l)$

$$
\left(\mathrm{R}^{2}=0,990, \mathrm{ER} T=\mathrm{I}, 84\right)
$$

Pour les autres constituants, les équations suivantes ont été obtenues : Protéines corporelles $(\mathrm{kg})=-2,494+0,055 \mathrm{PV}(\mathrm{kg})+0,2 \mathrm{II} \mathrm{ED}(l)$

$$
\left(\mathrm{R}^{2}=0,976 \mathrm{ETR}=0,6 \mathrm{I}\right)
$$

Minéraux corporels $(\mathrm{kg})=-0,002+0,057 \mathrm{ED}(\mathrm{kg})$

$$
(r=0,929, \mathrm{ETR}=0, \mathrm{I} 8)
$$

Energie corporelle $(\mathrm{MJ})=-66,6 \mathrm{I} 4+36,568 \mathrm{PV}(\mathrm{kg})-38,987 \mathrm{ED}(l)$

$$
\left(\mathrm{R}^{2}=0,993 \mathrm{ETR}=60,40\right)
$$

A partir de ces équations, nous avons estimé la composition corporelle de nos moutons. Les valeurs estimées et les valeurs réelles ont évidemment été le plus souvent très proches les unes des autres. Pour tester la valeur de cette estimation selon l'état d'engraissement des animaux, nous avons ici encore divisé nos moutons en deux groupes (gras et non gras, tab1. 3). Pour 1'eau et les graisses, les différences moyennes entre les valeurs réelles et estimées ont été :

\begin{tabular}{c|c|c}
\hline $\begin{array}{c}\text { Moutons } \\
\text { Sheep }\end{array}$ & $\begin{array}{c}\text { Eau (1) } \\
\text { Water }\end{array}$ & $\begin{array}{c}\text { Graisses (kg) } \\
\text { Fat }\end{array}$ \\
\hline Gras (Fat)...... . . . & $\begin{array}{r}0, \mathrm{I} 2 \pm \mathrm{I}, 74 \\
-0,08 \pm \mathrm{I}, 66\end{array}$ & $\begin{array}{c}0,30 \pm \mathrm{I}, 30 \\
0,3 \mathrm{I}+\mathrm{I}, 79\end{array}$ \\
\hline
\end{tabular}

I1 ne paraît y avoir aucune différence entre les groupes quant à la valeur de de l'estimation de l'eau et des graisses corporelles pour nos formules.

\section{Discussion}

Les techniques généralement utilisées pour la détermination de la composition chimique de l'organisme animal sont variées et plus ou moins fastidieuses : séparation des tissus mous et durs, broyage et mixage, congélation et pulvérisation. Pour notre part, nous avons retenu pour la préparation des échantillons des carcasses et des abats la tehnique de passage à l'autoclave mise au point à l'Université de Gent (DE WILDE, I977), Cette technique a pour avantage d'être de réalisation aisée et de nombreuses vérifications ont montré que les pertes de substances organiques sont négligeables.

L'engraissement d'un mouton adulte partant d'un poids moyen constitue essentiellement un dépôt de graisses (plus de 75 p. Ioo) avec un pourcentage de protéines inférieur à ro p. IOO (BURTON et al., I974, SykEs, I974). La comparaison de la composition chimique de nos animaux (gras et autres) nous permet d'arriver à la même conclusion. Chez des animaux de même race et de conformation zootechnique comparable, les changements de poids entraînent donc de nettes modifications de la composition corporelle. En conséquence, si le poids vide sans laine 
est connu, il est possible d'estimer la composition chimique des animaux avec une bonne approximation.

Dans nos conditions de travail, l'espace de diffusion de l'ean tritiée surestime en moyenne l'eat corporelle totale de $5,7 \mathrm{p}$. Ioo. Cette valeur est égale à celle obtenue par SEARLE (I970), inférieure à celles calculées par DONNELLY et FREER (I974), Farreil, et REARdon (I972), Foo', SkEdd et Mc Farlane (I979), KeEnan, Mc Manus et FreER (rg69). Foot, SkEEn et Mc FarLane (I979) dans une autre expérience et Sykes (I974) trouvent au contraire une bonne relation entre les deux paramètres.

Cette différence de 5,7 p. roo pouvait être attendue, car diverses sources d'erreur ne sont pas considérées par la technique. La perte d'eau tritiée par les urines atteint en moyenne $0,42 \mathrm{p}$. Ioo chez nos moutons en 7 heures; elle est de I,49 p. Ioo en 8 heures chez Smith et Sykes (I974). Les pertes cutanées et respiratoires seraient inférieures à I p. IOO ('TILL et Downes, mentionnés par SMITH et SxkEs, I974). Les échanges de tritium avec l'hydrogène corporel doivent aussi être mentionnés. Culabras et MOORE (I977) ont calculé que l'hydrogène échangeable total du corps animal représente $5,22 \mathrm{p}$. Ioo, mais ces auteurs concluent que ce maximum est très loin d'être atteint pendant les courtes périodes de temps utilisées pour la détermination de l'espace de diffusion de l'eau tritiée. FARRELL et REARDON (I972) estiment que 0,2 p. Ioo seulement de la dose injectée a servi aux échanges avec l'hydrogène des constituants corporels.

Une erreur dans l'estimation de l'eau corporelle pourrait aussi provenir du contenu digestif important du ruminant. Chez nos animaux, l'eau du contenu digestif représente en moyenne I7,9 p. Ioo de l'eau corporelle totale. Des valeurs entre 16 et 26 p. IOO ont été trouvées par d'autres auteurs (FARRELI. et REARDON, I972; Foot, Skedd et Mc Farlane, I979; KeEnan, Mc Manus et Freer, ig69; Sykes, r974). Dans nos conditions expérimentales nous attendons 7 heures après l'injection de l'eau tritiée pour effectuer la prise de sang destinée à calculer l'espace de diffusion de l'eau tritiée. Ce délai a été choisi parce que d'après les données de la littérature, il est suffisant pour assurer une diffusion uniforme de l'eau tritiée dans l'organisme et parce que, à ce moment, les pertes de tritium, notamment par les urines, ne sont pas encore très élevées.

Enfin, la non-prise en considération de la laine est une autre source possible d'erreur. Dans notre cas, en effet, l'eau de la laine représente en moyenne $0,5^{6} \mathrm{p}$. Ioo de 1'eau corporelle totale.

Nos valeurs montrent en plus que l'état d'engraissement de l'animal n'a pas d'effet apparent sur l'exactitude de l'estimation de l'eau corporelle par l'espace de diffusion. Il ne modifie pas non plus la valeur de l'estimation "in vivo " de la composition corporelle à partir du poids vif et de l'espace de diffusion. Nos formules peuvent donc être appliquées à des animaux adultes montrant une très grande variabilité pondérale.

L'estimation du poids des différents composants corporels de nos moutons a été réalisée à partir des équations décrites dans la littérature par les auteurs précités. Entre les valeurs estimées et les valeurs réelles, les différences vont de $+3,51$ (Foot, Skedd et Mc FArIane, I979) à - 6,81 (Houseman, Robinson et Fraser, I978) pour l'eau, de - 4,4 kg (SYKES, I974) à $+7,6 \mathrm{~kg}$ (DONNELLY et FREER, I974) pour les graisses. De grands écarts sont aussi enregistrés pour les protéines, les minéraux et l'énergie. De telles variations sont, comme l'écrit Sykes (I974), à mettre en relation avec le fait que, selon les études, l'éventail des variations du poids et de l'état d'engraissement des animaux est très variable. De toutes les équations considérées, celles de SEARLE (I970) donnent, avec nos animaux, les 
résultats les plus concordants. C'est aussi l'auteur qui, bien que travaillant avec des animaux en croissance-engraissement, utilisait les moutons montrant la plus grande variabilité (de 3 à $46 \mathrm{~kg}$ de poids vif, de 2,8 à $33,8 \mathrm{p}$. Ioo de graisses).

Dans nos conditions de travail, à savoir avec des moutons de race Suffolk de conformation moyenne, il nous est donc possible dorénavant de suivre chez des groupes d'animaux (3 ou 4 au moins par groupe) 1'évolution in vivo de la composition corporelle en fonction du degré d'engraissement.

Accepté pour publication en octobre 1979.

\section{Remerciements}

Ces recherches ont été réalisées sous les auspices de l'Institut pour l'Encouragement de la Recherche Scientifique dans 1'Industrie et l'Agriculture, rue de Crayer 6, B-Io5o Bruxelles. Nous remercions les Professeurs Elfins des Facultés de Namur et VANBFi iri et VERvack, de 1'Université de Louvain-la-Neuve, pour leur aide technique.

\section{Summary}

\section{Estimation of the chemical composition of adult sheep and variations according to feeding}

The purpose of the present study was to develop equations for estimating the chemical composition of adult sheep exhibiting highly variable weights and degrees of fatness. To that end $r_{2}$ ewes and 2 rams of the Suffolk breed, aged more than 2 I / 2 years were slaughtered and analysed after determination of the tritiated water space. Prior to slaughter the animals had been subjected to variable feeding conditions.

The live weight of the sheep ranged from 43.5 to $93.9 \mathrm{~kg}$ and the fat content of the wool free empty carcass, from 7.2 to 48.2 p. Ioo.

The weight difference between the fat animals (more than $60 \mathrm{~kg}$ wool free empty body weight) and the lean animals depended for $60 \mathrm{p}$. I oo on fat. When the wool free empty body weight is known it is possible to obtain an accurate estimation of the chemical body composition of the animals.

The tritiated water space overestimated by $5.7 \mathrm{p}$. I oo on an average the total body water volume. This difference was not affected by the degree of fatness of the animals. The following equations were calculated from live weight $(\mathrm{LW}, \mathrm{kg})$ and from the tritiated water space $(\mathrm{TOH}, \mathrm{I})$ :

$\begin{array}{llrl}\text { Total body water }(\mathrm{kg}) & = & 2.280+0.849 \mathrm{TOH} & (r=0,969) . \\ \text { Body fat }(\mathrm{kg}) & =-4.035+0.928 \mathrm{LW}-\mathrm{I} .022 \mathrm{TOH}\left(\mathrm{R}^{2}=0,990\right) . \\ \text { Body proteins }(\mathrm{kg}) & =-2.494+0.055 \mathrm{LW}+0.2 \mathrm{I} \mathrm{IOH}\left(\mathrm{R}^{2}=0,976\right) . \\ \text { Body minerals }(\mathrm{kg}) & =-0.022+0.057 \mathrm{TOH} & (r=0.929) . \\ \text { Body energy }(\mathrm{MJ}) & =-66.614+36.658 \mathrm{LW}-38.987 \mathrm{FD}\left(\mathrm{R}^{2}=0.993\right) .\end{array}$

These results show that it is possible to obtain a valid in vivo estimation of the chemical composition of adult sheep on the basis of live weight and tritiated water space.

\section{Références bibliographiques}

BLUNT, M. H., 1975. The blood of sheep, composition and function. Springer-Verlag, Berlit1Heidelberg-New York.

Burín J. H., Andirson M., REin J. T., I974. Some biological aspects of partial starvation. The effect of weight loss and regrowth on body composition in sheep. Br. $J . N u t^{\gamma}$., 32, 5I 5-527. 
CUIferras J. M., Moore F. D., I977. Total body water and the exchangeable hydrogen. I. Theoretical calculation of tonaqueous exchangeable hydrogen in man. Am. J. Physiol., 232, R54R59.

DE WILDE R., I977. Studie van een methode ter bepaling van eiwit- en energieretenties bij mestvarkens gebaseerd op karkasanalyse. Meded. Fac. Dievgeneesk. Rijksuniv. Gent, 20, Nrs 3-4.

DONNEILY J. R., FREFR M., I974. Prediction of body composition in live sheep. Aust. J. Agric. Res., 25, $825^{-8} 34$.

Dumon' B. L., I958. Méthodes indirectes de mesure de la graisse corporelle des mammifères. Cahiers techniques du centre de coordination des études et recherches sur la nutrition et l'alimentation. Cahier II. Centre National de la Recherche Scientifique. Paris.

FARRELL D. J., REARDON T. F., I972. Undernutrition in grazing sheep. III. Body composition and its estimation in vivo. Aust. J. Agric. Res., 23, 51 I-5I7.

FoOT J. Z., SKEDD E., McFarLaknE D. N., I979. Body composition in lactating sheep and its indirect measurement in the live animal using tritiated water. J. Agric. Sci., 92, 69-8I.

Houseman R. A., Robinson J. J., Fraser C., I978. The estimation of body water and fat in pregnant ewes deuterium oxide. Proc. Nutr. Soc., 37, 64 A.

KeENar D. M., McManus W. R., Frefr M., i 969 . Changes in the body composition and efficiency of mature sheep during loss and regain of live weight. J. Agric. Sci., Cam., 72, 139-147.

ROBELIN J., 1973. Estimation de la composition corporelle des animaux à partir des espaces de diffusion de 1'eau marquée. Ann. Biol. anim. Bioch. Biophys., 13, 285-305.

SEARLE T. W., I970. Body composition in lambs and young sheep and its prediction in vivo from tritiated water space and body weight. J. Agric. Sci., Camb., 74, 357-362.

SMith B. S. W., Sykes A. R., I 974 . The effect of route of dosing and method of estimation of tritiated water space on the determination of total body water and the prediction of body fat in sheep. J. Agric. Sci., Camb., 82, $105^{-1} 12$.

Sykes A. R., I974. The prediction of the body composition of hill sheep from body weight, red cell volume and tritiated water space. $J$. A gric. Sci., Camb., 82, 269-275. 